\title{
On a possibility of phase transitions in the geometric structure of space-time ${ }^{1}$
}

\author{
G.Yu. Bogoslovsky 自 \\ Institute of Nuclear Physics, Moscow State University, 119899 Moscow, Russia \\ H.F. Goenner 角 \\ Institute for Theoretical Physics, University of Göttingen, D-37073, Göttingen, Germany
}

\begin{abstract}
It is shown that space-time may be not only in a state which is described by Riemann geometry but also in states which are described by Finsler geometry. Transitions between various metric states of space-time have the meaning of phase transitions in its geometric structure. These transitions together with the evolution of each of the possible metric states make up the general picture of space-time manifold dynamics.

PACS : 95.30.-k; 95.30.Sf; 98.80.Bp; 98.80.Hw; 02.40.-k

Keywords: Riemannian and Finslerian space-time; Phase transitions;

Relativistic cosmology
\end{abstract}

According to the contemporary cosmological models [1], space-time is Riemannian, i.e. locally isotropic, and preserves its local isotropy in the process of the Universe evolution. At the same time there exist some indirect indications of the fact that nowadays spacetime has a weak relic local anisotropy and therefore it is not unlikely that it is described by Finsler geometry [2] rather than by Riemann geometry. Moreover, if it appears that the Hubble constant has no dipole anisotropy correlated with the dipole anisotropy of the microwave background radiation then it will unequivocally indicate a strong local anisotropy of space-time at the early stage of the Universe evolution.

One of the possible mechanisms of the appearance of a local anisotropy in space-time is the induced phase transition in its geometric structure, caused by the breakdown of higher gauge symmetries and by the appearance of masses in fundamental fields of matter. This involves changes in the metric properties of space-time manifold and it goes over from a state described by Riemann geometry into a state described by Finsler geometry. Since Finslerian space-time differs from Riemannian space-time by the anisotropy of its tangent spaces, in such a transition there occurs a flagrant violation of the local Lorentz

\footnotetext{
${ }^{1}$ This research is supported by DFG grant No.7/25/94 and INTAS-93-1630-EXT grant

${ }^{2}$ E-mail: bogoslov@theory.npi.msu.su

${ }^{3}$ E-mail: goenner@theorie.physik.uni-goettingen.de
} 
symmetry of space-time. In the course of subsequent expansion of the Universe the initial strong local anisotropy of the Finslerian space-time monotonically decreases and, on the average, tends to zero together with its curvature. Gradually the local Lorentz symmetry of space-time is also restored.

The first indication of the fact that nowadays the local Lorenz symmetry still remains slightly broken was obtained from the investigation of the spectrum of primary cosmic superhigh-energy protons. The point is that according to the calculations $[3,4]$, which substantially employ the local Lorentz symmetry of space-time, the proton energy spectrum should be cut off (due to the intense production of pions on relic radiation photons ) at proton energies $\sim 5 \times 10^{19} \mathrm{eV}$. The experimental data [5,6], however, are most likely indicative of the absence of such an effect. This situation induced the investigators $[7,8]$ to assume that the conventional Lorentz transformations become invalid for the Lorentz factors $\gamma>5 \times 10^{10}$ and the correct relation between the various inertial reference frames at any values of $\gamma$ is provided by the other, so-called generalized Lorentz transformations. Subsequently [9] it has indeed become possible to find the generalized Lorentz transformations. It appeared that they belong to a group of local relativistic symmetry of Finslerian space-time, in which case the smaller the local anisotropy of Finslerian space-time, i.e. the closer is it to Riemannian one, the closer to the velocity of light tend the generalized Lorentz transformations to be markedly different from the conventional ones. Therefore the use of these transformations in calculating the cutoff point of the primary cosmic proton spectrum enables one, in principle, to remove the emerged discrepancy between the theoretical predictions and the experimental data pertaining to the superhigh energy region.

In order to demonstrate that the existence of the generalized Lorentz transformations necessarily leads to the existence of a local anisotropy in space-time, first consider a twodimensional event space. In this case the generalized Lorentz transformations appear as

$$
\left\{\begin{array}{l}
x_{0}^{\prime}=e^{-r \alpha} \quad\left(\begin{array}{r}
\left.x_{0} \cosh \alpha-x \sinh \alpha\right) \\
x^{\prime}=e^{-r \alpha}
\end{array}\left(-x_{0} \sinh \alpha+x \cosh \alpha\right),\right.
\end{array}\right.
$$

where $\tanh \alpha=v / c$ and $r$ is the dimensionless parameter of the scale transformation. It is obvious that in spite of the presence of additional dilatation the transformations (1) still remain linear, constitute a group with the group parameter $\alpha$ and lead to Einstein's law of velocity addition. However, the pseudo-Euclidean metric is no longer their invariant. It is easy to verify that the invariant of the transformations (1) is the metric

$$
d s^{2}=\left[\frac{\left(d x_{0}-d x\right)^{2}}{d x_{0}^{2}-d x^{2}}\right]^{r}\left(d x_{0}^{2}-d x^{2}\right) .
$$

The given metric belongs to a class of Finslerian metrics and describes a flat but anisotropic space of events. While we consider a two-dimensional anisotropic space, its anisotropy manifests itself in noninvariance of the metric (2) under the reflection transformation $x_{0} \rightarrow-x_{0}$ or $x \rightarrow-x$. The anisotropy just mentioned disappears only in the case $r=0$, when the event space becomes pseudo-Euclidean and the generalized Lorentz transformations (1) become the conventional Lorentz ones. Therefore the parameter $r$ characterizes the value of the space anisotropy. 
If in (2) we replace the forms $\left(d x_{0}^{2}-d x^{2}\right)$ and $\left(d x_{0}-d x\right)$ by their four-dimensional analogs, i.e. make the substitution

$$
\left(d x_{0}^{2}-d x^{2}\right) \rightarrow\left(d x_{0}^{2}-d \vec{x}^{2}\right) ; \quad\left(d x_{0}-d x\right) \rightarrow\left(d x_{0}-\vec{\nu} d \vec{x}\right)
$$

then we can thus obtain the corresponding four-dimensional metric

$$
d s^{2}=\left[\frac{\left(d x_{0}-\vec{\nu} d \vec{x}\right)^{2}}{d x_{0}^{2}-d \vec{x}^{2}}\right]^{r}\left(d x_{0}^{2}-d \vec{x}^{2}\right) .
$$

The Finslerian metric (3) describes a flat anisotropic space-time with partially broken rotational symmetry. This signifies that instead of the 3-parameter rotation group, which was admitted by the isotropic pseudo-Euclidean event space, the space-time (3) admits only a 1-parameter group of rotations about the unit vector $\vec{\nu}$ which indicates the preferred direction in a 3 -space. As a result the homogeneous isometry group of the space-time (3) turns out to be a 4-parameter group [9] rather than a 6-parameter group, in which case as the transformations of relativistic symmetry it incorporates a 3-parameter noncompact subgroup of the generalized Lorentz transformations

$$
x^{i}=D(\vec{v}, \vec{\nu}) R_{j}^{i}(\vec{v}, \vec{\nu}) L_{k}^{j}(\vec{v}) x^{k},
$$

where $L_{k}^{j}(\vec{v})$ is a conventional Lorentz boost, $R_{j}^{i}(\vec{v}, \vec{\nu})$ is the rotation of the space axes about the vector $[\vec{v} \vec{\nu}]$ through an angle determined by relativistic aberration of the preferred direction $\vec{\nu}, D(\vec{v}, \vec{\nu})=\left[(1-\vec{v} \vec{\nu} / c) /{\sqrt{1-\vec{v}^{2} / c^{2}}}^{r} I\right.$, and $I$ is the unit matrix.

The difference of the metric (3) from the pseudo-Euclidean one is such that it does not involve the light cone equation. Therefore the 3-geometry still remains Euclidean. At the same time the anisotropy of the event space (3) leads to nontrivial consequences even at the level of nonrelativistic physics. In particular [10], the effective inertness of a particle of mass $m$ turns out to be dependent on the quantities $r$ and $\vec{\nu}$, which characterize space anisotropy, and is determined by a tensor of inert mass

$$
\mathfrak{M}_{\alpha \beta}=m(1-r)\left(\delta_{\alpha \beta}+r \nu_{\alpha} \nu_{\beta}\right)
$$

Thus Newton's second law takes the form $\mathfrak{M}_{\alpha \beta} a^{\beta}=F_{\alpha}$.

It should be noted here that the parameters $r$ and $\vec{\nu}$ are in fact local values of the corresponding fields $r(x)$ and $\nu_{i}(x)$, in which case $\nu_{i} \nu^{i}=0$. Together with the field of the Riemannian metric tensor $g_{i k}(x)$, responsible for gravitation, these fields determine the Finslerian metric of a curved locally anisotropic space-time

$$
d s^{2}=\left[\frac{\left(\nu_{i} d x^{i}\right)^{2}}{g_{i k} d x^{i} d x^{k}}\right]^{r}\left(g_{i k} d x^{i} d x^{k}\right) .
$$

This space-time has flat anisotropic spaces (3) as tangent spaces, possesses the 3parameter group of local relativistic symmetry (4) and reduces to the Riemannian spacetime at $r(x)=0$. Noteworthy is also the fact that the dynamics of the Finsler space (6) is completely determined by the dynamics of the fields $g_{i k}(x), r(x)$ and $\nu_{i}(x)$ and is 
described by a system of field equations [11-13] generalizing the Einstein equations. In this case, within the framework of the Finslerian theory of gravitation, the particle inert mass turns out, according to (5), to be a tensor field on space-time and is ultimately determined (in accordance with the Mach principle) by the distribution and motion of external matter. The possibility of realizing the Mach principle is characteristic of the Finslerian theory. In the metric theories of gravitation, employing the Riemannian model of space-time, there is no such possibility since there is no local anisotropy field itself, i.e. $r(x)=0$.

Obviously, the maximum permissible local value of the field $r(x)$ is a value of $r=1$. At such $r$ the linear element of the tangent space (3) degenerates into the total differential

$$
d s=d x_{0}-\vec{\nu} d \vec{x}
$$

and, consequently, the action $S=-m c \int_{a}^{b} d s$ for a free particle of mass $m$ is no longer dependent on the shape of the world line connecting the points $a$ and $b$. This means that at $r=1$ any massive particle loses its inertness. The aforesaid is illustrated by (5) in accordance with which $\mathfrak{M}_{\alpha \beta}=0$ at $r=1$. At $r=1$, along with inertness the notion of spatial extension disappeares, which is due to the absence of a light cone in this case and, consequently, of the possibility itself of determining spatial distances using the exchange of light signals. As a result, in the space-time (7) there remains the single physical characteristic, namely, time duration and it should be regarded as an interval of absolute time.

Since the "metric" (7) is a special case of the metric (3), then to within isomorphism all the transformations, which leave invariant the metric (3), leave invariant the "metric" (7) as well. At the same time, on making the substitution of the variables $\nu_{1} x_{1} \rightarrow$ $x_{1}, \nu_{2} x_{2} \rightarrow x_{2}, \nu_{3} x_{3} \rightarrow x_{3} ; \nu_{1}, \nu_{2}, \nu_{3} \neq 0$ and on representing (7) as

$$
d s=d x_{0}-d x_{1}-d x_{2}-d x_{3},
$$

one can find that in comparison with (3) the "metric" (7) has an additional Abelian 3parameter isometry group. It turned out [14] that there exists a homogeneous noncompact group which to within isomorphism coincides with the above-mentioned Abelian group and is a group of relativistic symmetry of a flat space-time with the metric

$$
\begin{aligned}
d s & =\left(d x_{0}-d x_{1}-d x_{2}-d x_{3}\right)^{\left(1+r_{1}+r_{2}+r_{3}\right) / 4}\left(d x_{0}-d x_{1}+d x_{2}+d x_{3}\right)^{\left(1+r_{1}-r_{2}-r_{3}\right) / 4} \\
& \times\left(d x_{0}+d x_{1}-d x_{2}+d x_{3}\right)^{\left(1-r_{1}+r_{2}-r_{3}\right) / 4}\left(d x_{0}+d x_{1}+d x_{2}-d x_{3}\right)^{\left(1-r_{1}-r_{2}+r_{3}\right) / 4}
\end{aligned} .
$$

The given Finslerian metric depends on three parameters $r_{1}, r_{2}$ and $r_{3}$ and describes an anisotropic space-time with the entirely broken symmetry with respect to the rotation group. The permissible values of the parameters $r_{1}, r_{2}$ and $r_{3}$ are limited by the conditions

$$
\begin{array}{ll}
1+r_{1}+r_{2}+r_{3}>0, & 1+r_{1}-r_{2}-r_{3}>0, \\
1-r_{1}+r_{2}-r_{3}>0, & 1-r_{1}-r_{2}+r_{3}>0,
\end{array}
$$

which ensure the fact that the section of a light cone by hyperplane $d x_{0}=$ const is a closed convex surface, and this ensures the applicability of the procedure of exchange of light signals for determining 3-space distances. 
In the limiting case, where, for example, $r_{1}=r_{2}=r_{3}=1$, the metric (9) degenerates into a 1-form (8), i.e. into the total differential of absolute time. If we now recall that the metric (3) of the flat anisotropic space-time with the partially broken 3-rotational symmetry also degenerates at $r=1$ into the total differential of absolute time, then it suggests that absolute time is not a stable degenerate state of space-time and as a result of the "primary" phase transition it may turn either into the partially anisotropic space-time (3) or into the entirely anisotropic space-time (9). In any case such a phase transition is accompanied by an "act of creation" of a three-dimensional space, in which case its geometry depends on the direction of the phase transition. In the passage to (3) there occurs a 3-space with locally Euclidean geometry while in the passage to (9) there occurs, as will be shown below, a 3-space with locally non-Euclidean geometry. Thus it is precisely absolute time that is a connecting link by which the principle of correspondence is satisfied for the Finsler spaces (3) and (9).

It has already been pointed out that the homogeneous isometry group of the event space (9) is an Abelian 3-parameter noncompact group. The transformations belonging to it link various inertial reference frames and are of the form

$$
x_{i}^{\prime}=D L_{i k} x_{k}
$$

where: $D=\exp \left(-r_{1} \alpha_{1}-r_{2} \alpha_{2}-r_{3} \alpha_{3}\right) ;$ the matrices

$$
L_{i k}=\left(\begin{array}{rrrr}
\mathcal{A} & -\mathcal{B} & -\mathcal{C} & -\mathcal{D} \\
-\mathcal{B} & \mathcal{A} & \mathcal{D} & \mathcal{C} \\
-\mathcal{C} & \mathcal{D} & \mathcal{A} & \mathcal{B} \\
-\mathcal{D} & \mathcal{C} & \mathcal{B} & \mathcal{A}
\end{array}\right)
$$

are unimodular, whereby

$$
\begin{aligned}
& \mathcal{A}=\cosh \alpha_{1} \cosh \alpha_{2} \cosh \alpha_{3}+\sinh \alpha_{1} \sinh \alpha_{2} \sinh \alpha_{3}, \\
& \mathcal{B}=\cosh \alpha_{1} \sinh \alpha_{2} \sinh \alpha_{3}+\sinh \alpha_{1} \cosh \alpha_{2} \cosh \alpha_{3}, \\
& \mathcal{C}=\cosh \alpha_{1} \sinh \alpha_{2} \cosh \alpha_{3}+\sinh \alpha_{1} \cosh \alpha_{2} \sinh \alpha_{3}, \\
& \mathcal{D}=\cosh \alpha_{1} \cosh \alpha_{2} \sinh \alpha_{3}+\sinh \alpha_{1} \sinh \alpha_{2} \cosh \alpha_{3}
\end{aligned}
$$

and $\alpha_{1}, \alpha_{2}$, and $\alpha_{3}$ are group parameters.

In place of $\alpha_{1}, \alpha_{2}$, and $\alpha_{3}$ as group parameters we can use the velocity components $v_{1}, v_{2}$, and $v_{3}$ of the primed reference frame. For this purpose it is sufficient to put $x_{1}^{\prime}=x_{2}^{\prime}=x_{3}^{\prime}=0$ in the transformations inverse to (10). As a result we arrive at the relations

$$
\begin{aligned}
& v_{1}=\left(\tanh \alpha_{1}-\tanh \alpha_{2} \tanh \alpha_{3}\right) /\left(1-\tanh \alpha_{1} \tanh \alpha_{2} \tanh \alpha_{3}\right), \\
& v_{2}=\left(\tanh \alpha_{2}-\tanh \alpha_{1} \tanh \alpha_{3}\right) /\left(1-\tanh \alpha_{1} \tanh \alpha_{2} \tanh \alpha_{3}\right), \\
& v_{3}=\left(\tanh \alpha_{3}-\tanh \alpha_{1} \tanh \alpha_{2}\right) /\left(1-\tanh \alpha_{1} \tanh \alpha_{2} \tanh \alpha_{3}\right) .
\end{aligned}
$$

The inverse relations appear as 


$$
\begin{aligned}
& \alpha_{1}=\frac{1}{4} \ln \frac{\left(1+v_{1}-v_{2}+v_{3}\right)\left(1+v_{1}+v_{2}-v_{3}\right)}{\left(1-v_{1}-v_{2}-v_{3}\right)\left(1-v_{1}+v_{2}+v_{3}\right)}, \\
& \alpha_{2}=\frac{1}{4} \ln \frac{\left(1-v_{1}+v_{2}+v_{3}\right)\left(1+v_{1}+v_{2}-v_{3}\right)}{\left(1-v_{1}-v_{2}-v_{3}\right)\left(1+v_{1}-v_{2}+v_{3}\right)}, \\
& \alpha_{3}=\frac{1}{4} \ln \frac{\left(1-v_{1}+v_{2}+v_{3}\right)\left(1+v_{1}-v_{2}+v_{3}\right)}{\left(1-v_{1}-v_{2}-v_{3}\right)\left(1+v_{1}+v_{2}-v_{3}\right)} .
\end{aligned}
$$

Noteworthy is the fact that such an observable as, for example, the velocity $v$ is no longer determined now by the formula $v=\sqrt{v_{1}^{2}+v_{2}^{2}+v_{3}^{2}}$. This comes from the fact that in the case of the flat event space (9) the geometry of the corresponding 3-space turns out to be non-Euclidean while time $x_{0}$, to be coordinate time only.

In order to determine how the difference of coordinates of two events in the event space (9) is related to observables and thereby to obtain correct formulas for the observables it is necessary to use a procedure involving the exchange of light signals between neighbouring points of the 3-space. For this purpose we first of all note that according to the definition (9) the tolerance range of $d x_{i}$ values is limited by the conditions

$$
\left\{\begin{array}{l}
d x_{0}-d x_{1}-d x_{2}-d x_{3} \geq 0 \\
d x_{0}-d x_{1}+d x_{2}+d x_{3} \geq 0 \\
d x_{0}+d x_{1}-d x_{2}+d x_{3} \geq 0 \\
d x_{0}+d x_{1}+d x_{2}-d x_{3} \geq 0
\end{array}\right.
$$

These conditions determine either the timelike interval between two events or the interval equal to zero and are invariant under the relativistic transformations (10). Apart from this the transformations (10) leave invariant the sign of $d x_{0}$. Let now $d x_{0}>0$. Then in terms of the component $v_{\alpha}=d x_{\alpha} / d x_{0}$ of the coordinate velocity the conditions (11) can be rewritten as follows

$$
\left\{\begin{array}{l}
1-v_{1}-v_{2}-v_{3} \geq 0 \\
1-v_{1}+v_{2}+v_{3} \geq 0 \\
1+v_{1}-v_{2}+v_{3} \geq 0 \\
1+v_{1}+v_{2}-v_{3} \geq 0
\end{array} .\right.
$$

The range of $v_{\alpha}$ values, limited by these conditions, is depicted in Figure 1 . The range considered represents a regular tetrahedron with the center at the origin of a rectangular system of the coordinates $v_{1}, v_{2}, v_{3}$. The velocities corresponding to the timelike intervals $d s$ fill the inner region of the tetrahedron while the velocities, which describe the propagation of light signals and ensure the fulfillment of the equality $d s=0$, fill the surface of the tetrahedron. Figure 1, next to their letterings, gives the coordinates of eight of the fourteen characteristic points which lie on the tetrahedron surface. The coordinates of the remaining six points are as follows $\varepsilon(0,-1,0) ; \beta(0,1,0) ; \Gamma(1 / 3,1 / 3,1 / 3) ; \Theta(-1 / 3,-1 / 3,1 / 3) ; \Phi(-1 / 3,1 / 3,-1 / 3) ;$ $\Omega(1 / 3,-1 / 3,-1 / 3)$. The role of these points consists in that with the aid of them the tetrahedron surface is divided into 12 equal tetragons and they, in turn, are grouped 
into six pairs of mutually conjugated (with respect to a reflection operation at the origin ) tetragons. By labelling the reflection operation with a symbol $\longleftrightarrow$ we obtain the following pairs

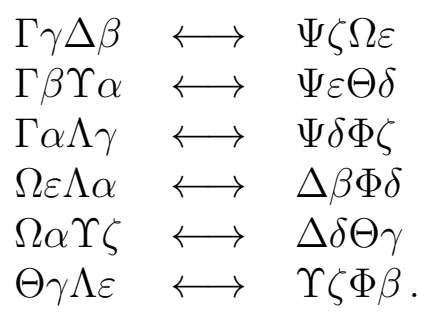

Consider, for example, a tetragon $\Gamma \gamma \Delta \beta$. The points filling it determine the coordinate velocities of light signals propagating within a solid angle ( sector) $\Gamma \gamma \Delta \beta o$. Rays propagating in the opposite directions belong to the sector $\Psi \zeta \Omega \varepsilon O$ while the velocities of light, corresponding to them, fill the tetragon $\Psi \zeta \Omega \varepsilon$. If $\left(v_{1}, v_{2}, v_{3}\right)$ are the velocity components of an initial light signal propagating in the sector $\Gamma \gamma \Delta \beta O$ and $\left(\tilde{v}_{1}, \tilde{v}_{2}, \tilde{v}_{3}\right)$ are the velocity components of the reflected signal, i.e. the signal belonging to the sector $\Psi \zeta \Omega \varepsilon o$, then, as can readily be verified,

$$
\tilde{v}_{1}=-\frac{v_{1}}{v_{2}+v_{3}-v_{1}} ; \quad \tilde{v}_{2}=-\frac{v_{2}}{v_{2}+v_{3}-v_{1}} ; \quad \tilde{v}_{3}=-\frac{v_{3}}{v_{2}+v_{3}-v_{1}}
$$

Let now $(0,0,0,0)$ be the coordinates of the event associated with the emission of a light signal within the sector $\Gamma \gamma \Delta \beta o$ and $\left(d x_{0}^{(1)}, d x_{1}, d x_{2}, d x_{3}\right)$ are the coordinates of the event associated with the reflection of the given signal. In this case $\left(d x_{0}^{(1)}+d x_{0}^{(2)}, 0,0,0\right)$ are the coordinates of the event involving the return of the signal to the initial point. Then the coordinate velocities of the initial and reflected signals can be represented, respectively, as

$$
\begin{gathered}
v_{1}=d x_{1} / d x_{0}^{(1)}, v_{2}=d x_{2} / d x_{0}^{(1)}, v_{3}=d x_{3} / d x_{0}^{(1)} \\
\tilde{v}_{1}=-d x_{1} / d x_{0}^{(2)}, \tilde{v}_{2}=-d x_{2} / d x_{0}^{(2)}, \tilde{v}_{3}=-d x_{3} / d x_{0}^{(2)} .
\end{gathered}
$$

It follows from $(13),(14)$ and (12) that

$$
d x_{0}^{(2)} / d x_{0}^{(1)}=v_{2}+v_{3}-v_{1} .
$$

Since the initial ray is prescribed within the sector $\Gamma \gamma \Delta \beta o$ and the tetragon $\Gamma \gamma \Delta \beta$ belongs to the facet $\Delta \Upsilon \Lambda$ then the components $v_{\alpha}$ satisfy the equation

$$
1-v_{1}-v_{2}-v_{3}=0
$$

Using (15) and (16) and taking into account the definition (13), we thus arrive at the relations

$$
\begin{gathered}
\left(d x_{0}^{(1)}+d x_{0}^{(2)}\right) / 2=d x_{2}+d x_{3}, \\
\left(d x_{0}^{(1)}-d x_{0}^{(2)}\right) / 2=d x_{1} .
\end{gathered}
$$


By its meaning formula (17) determines within the sector $\Gamma \gamma \Delta \beta o$ the 3 -space distance between the points $(0,0,0)$ and $\left(d x_{1}, d x_{2}, d x_{3}\right)$, that is, it determines the 3 -metric in the form

$$
d l=d x_{2}+d x_{3} .
$$

According to the definition of the coordinates of events, at the instant of signal reflection a coordinate clock, placed at the reflection point, indicates the time $d x_{0}^{(1)}$ and a coordinate clock, placed at the point of signal emission, indicates the time $\left(d x_{0}^{(1)}+d x_{0}^{(2)}\right) / 2$. These indications differ by the value

$$
\Delta x_{0}=d x_{0}^{(1)}-\frac{d x_{0}^{(1)}+d x_{0}^{(2)}}{2}=\frac{d x_{0}^{(1)}-d x_{0}^{(2)}}{2} .
$$

Therefore, taking into account the relation (18), the coordinate clocks within the sector $\Gamma \gamma \Delta \beta o$ are synchronized using the following algorithm

$$
\Delta x_{0}=d x_{1}
$$

Finally determine the observable value $v$ of the particle velocity within the sector $\Gamma \gamma \Delta \beta o$. Assume that at the instant of starting of a particle from the point with the space coordinates $(0,0,0)$ the first clock placed at this point indicated the time 0 while at the instant of its arrival at the point $\left(d x_{1}, d x_{2}, d x_{3}\right)$ the second clock present there indicates the time $d x_{0}$. Since, according to (20), at the instant of particle starting the second clock indicated the time $\Delta x_{0}=d x_{1}$ rather than the time 0 , the true time interval spent on the displacement $d \vec{x}$ is $d \tau=\left(d x_{0}-d x_{1}\right)$ rather than $d x_{0}$. Considering now that the length of the vector $d \vec{x}$ is calculated with the aid of (19), we conclude that

$$
v=\frac{d l}{d \tau}=\frac{d x_{2}+d x_{3}}{d x_{0}-d x_{1}}=\frac{v_{2}+v_{3}}{1-v_{1}}
$$

According to $(21), v \leq 1$, in which case for a photon $v=1$. In the latter case $(21)$ is equivalent to (16) and, hence, $d s=0$.

Having made similar calculations we can determine the observables $d l, \Delta x_{0}$ and $v$ for each of the remaining eleven sectors. The complete set of the corresponding formulas is presented in the Table of observables. Using this Table we demonstrate what, for example, an Euclidean image of the non-Euclidean sphere of radius $d l$ is. For this purpose let us introduce a rectangular system of coordinates $d x_{1}, d x_{2}, d x_{3}$ in the Euclidean 3-space. One can readily see that each of the twelve sectors cuts its own piece (a rhomb) out of the corresponding plane $d l=$ const. All the twelve rhombs turn out to be equal to each other and they together make up the surface of a regular rhombic dodecahedron. Such a dodecahedron is depicted in Figure 2 . In fact it represents the indicatrix of a flat 3 -space whose symmetry is determined by a group of the corresponding discrete transformations rather than by the rotation group.

Having considered, along with the locally isotropic Riemannian metric, Finslerian metrics with the partially (3) and entirely (9) broken local isotropy, as a result we have obtained their unified description. It is generally agreed that the evolution of the Universe 
occurs within the framework of the Riemannian geometric model of space-time. At the same time our results [15] indicate that space-time may be not only in a state which is described by Riemann geometry but also in states which are described by Finsler geometry. Transitions between various metric states of space-time have the meaning of phase transitions in its geometric structure. These transitions together with the evolution of each of the possible metric states make up the general picture of space-time manifold dynamics.

In conclusion we note that apart from the Riemannian metric only two types of Finslerian metrics, namely, the metrics (3) and (9), admit 3-parameter groups of local relativistic symmetry. Therefore within the framework of classical theory the three cited metrics make up the complete ensemble of the possible metric states of space-time manifold. In the corresponding quantum theory this ensemble is substantially expanded to admit the inclusion of Finslerian metrics [16] with partially broken local relativistic symmetry and also of Finslerian metrics $[17,18]$ which describe Berwald spaces with entirely broken local relativistic symmetry.

\section{References}

[1] H. Goenner, Einführung in die Kosmologie (Spektrum, Akad. Verl., Berlin, 1994).

[2] H. Rund, The Differential Geometry of Finsler Spaces (Springer-Verl., BerlinGöttingen-Heidelberg, 1959 ) .

[3] K. Greisen, Phys. Rev. Lett. 16 ( 1966$) 748$.

[4] G.T. Zatsepin, V.A. Kuz'min, Pis'ma Zh. Eksper. Teor. Fiz. 4 ( 1966 ) 114.

[5] G.B. Khristiansen, Cosmic Rays of Superhigh Energies (Moscow University Press, Moscow, 1974), [in Russian].

[6] T. Doi et al., in: Proc. 24th Int. Cosmic Ray Conf. 2 (Roma 1995) 740 .

[7] D.A. Kirzhnits, V.A. Chechin, Pis'ma Zh. Eksper. Teor. Fiz. 14 (1971) 261.

[8] D.A. Kirzhnits, V.A. Chechin, Yadern. Fiz. 15 ( 1972 ) 1051.

[9] G.Yu. Bogoslovsky, Nuovo Cim. B 40 ( 1977 ) 99; B 43 ( 1978 ) 377.

[10] G.Yu. Bogoslovsky, Nuovo Cim. B 77 ( 1983 ) 181.

[11] G.Yu. Bogoslovsky, Theory of Locally Anisotropic Space-Time (Moscow University Press, Moscow, 1992 ), [in Russian] .

[12] G.Yu. Bogoslovsky, Class. Quantum Grav. 9 ( 1992 ) 569.

[13] G.Yu. Bogoslovsky, Fortschr. Phys. 42 (1994) 143; Phys. Part. Nucl. 24 (1993) 354 . 
[14] G.Yu. Bogoslovsky, H.F. Goenner, in: Abstr. 5th Int. Wigner Symposium (Vienna 1997) 20 .

[15] H.F. Goenner, G.Yu. Bogoslovsky, Preprint NPI MSU-97-29/480 .

[16] H.F. Goenner, G.Yu. Bogoslovsky, Preprint NPI MSU-96-35/442 (gr-qc/9701067) .

[17] R.K. Tavakol, N. Van den Bergh, Phys. Lett. A 112 ( 1985$) 23$.

[18] R.K. Tavakol, N. Van den Bergh, GRG 18 ( 1986 ) 849.

\section{Figure and Table captions}

Fig. 1 : The relativistically invariant range of permissible $v_{\alpha}$ values.

Fig. 2: A regular rhombic dodecahedron as an Euclidean image of the sphere of radius $d l$, prescribed in the flat non-Euclidean 3-space.

Tabl. : Table of observables. 


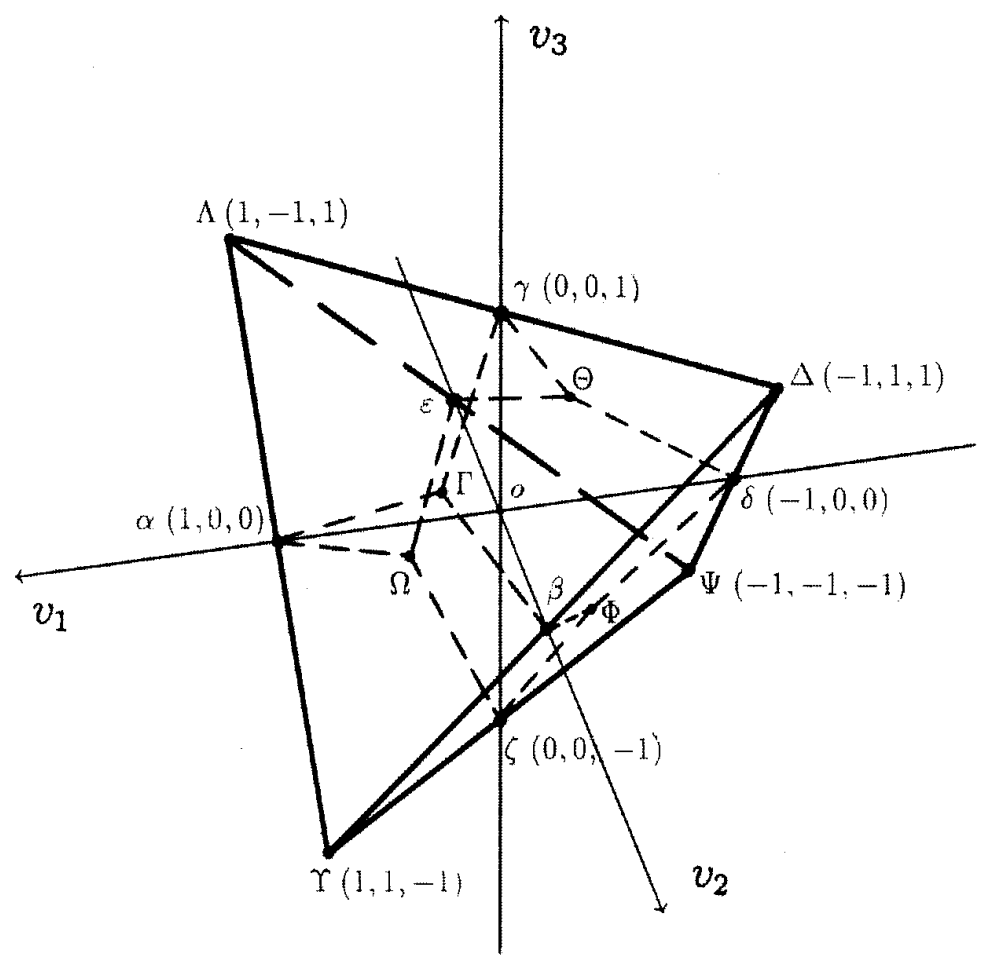

Figure 1: The relativistically invariant range of permissible $v_{\alpha}$ values. 


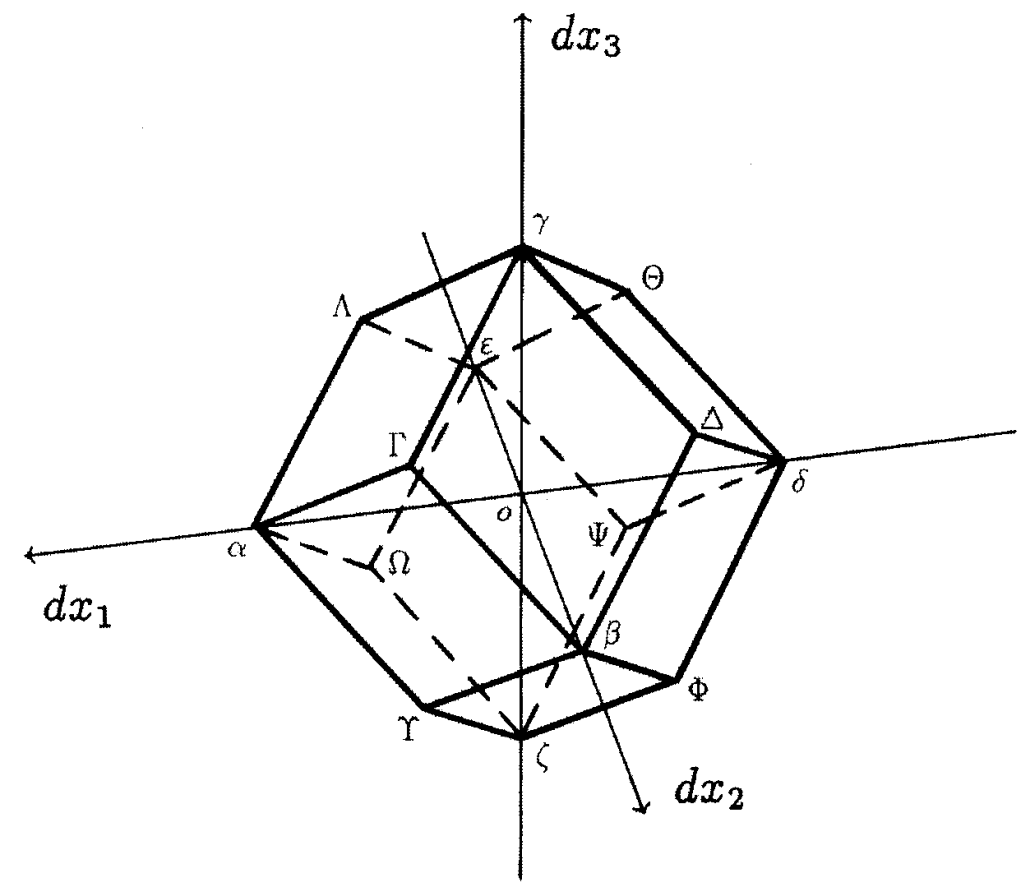

Figure 2: A regular rhombic dodecahedron as an Euclidean image of the sphere of radius $d l$, prescribed in the flat non-Euclidean 3-space. 
Table of observables

\begin{tabular}{|cc|c|c|c|}
\hline \multicolumn{2}{|c|}{ sector } & $d l$ & $\Delta x_{0}$ & $v$ \\
\hline \hline$\Gamma \gamma \Delta \beta o$ & & $d x_{2}+d x_{3}$ & $d x_{1}$ & $\left(v_{2}+v_{3}\right) /\left(1-v_{1}\right)$ \\
\hline & $\Psi \zeta \Omega \varepsilon o$ & $-\left(d x_{2}+d x_{3}\right)$ & $d x_{1}$ & $-\left(v_{2}+v_{3}\right) /\left(1-v_{1}\right)$ \\
\hline$\Gamma \beta \Upsilon \alpha o$ & & $d x_{1}+d x_{2}$ & $d x_{3}$ & $\left(v_{1}+v_{2}\right) /\left(1-v_{3}\right)$ \\
\hline & $\Psi \varepsilon \Theta \delta o$ & $-\left(d x_{1}+d x_{2}\right)$ & $d x_{3}$ & $-\left(v_{1}+v_{2}\right) /\left(1-v_{3}\right)$ \\
\hline$\Gamma \alpha \Lambda \gamma o$ & & $d x_{1}+d x_{3}$ & $d x_{2}$ & $\left(v_{1}+v_{3}\right) /\left(1-v_{2}\right)$ \\
\hline \multicolumn{2}{|c|}{$\Psi \delta \Phi \zeta o$} & $-\left(d x_{1}+d x_{3}\right)$ & $d x_{2}$ & $-\left(v_{1}+v_{3}\right) /\left(1-v_{2}\right)$ \\
\hline$\Omega \varepsilon \Lambda \alpha o$ & & $d x_{1}-d x_{2}$ & $-d x_{3}$ & $\left(v_{1}-v_{2}\right) /\left(1+v_{3}\right)$ \\
\hline \multicolumn{2}{|c|}{$\Delta \beta \Phi \delta o$} & $-\left(d x_{1}-d x_{2}\right)$ & $-d x_{3}$ & $-\left(v_{1}-v_{2}\right) /\left(1+v_{3}\right)$ \\
\hline$\Omega \alpha \Upsilon \zeta o$ & & $d x_{1}-d x_{3}$ & $-d x_{2}$ & $\left(v_{1}-v_{3}\right) /\left(1+v_{2}\right)$ \\
\hline \multicolumn{2}{|r|}{$\Delta \delta \Theta \gamma o$} & $-\left(d x_{1}-d x_{3}\right)$ & $-d x_{2}$ & $-\left(v_{1}-v_{3}\right) /\left(1+v_{2}\right)$ \\
\hline$\Theta \gamma \Lambda \varepsilon o$ & & $d x_{3}-d x_{2}$ & $-d x_{1}$ & $\left(v_{3}-v_{2}\right) /\left(1+v_{1}\right)$ \\
\hline \multicolumn{2}{|r|}{$\Upsilon \zeta \Phi \beta o$} & $-\left(d x_{3}-d x_{2}\right)$ & $-d x_{1}$ & $-\left(v_{3}-v_{2}\right) /\left(1+v_{1}\right)$ \\
\hline
\end{tabular}

